\title{
Biochemical and Immunological Predictors of Non-healing in Individuals with Early-stage Diabetic Foot Ulcers
}

\author{
Jayashree Vijaya Raghavan ${ }^{1}$, Shruthi Ksheera Sagar ${ }^{1}$, Vinod Kumar Dorai ${ }^{1,2}$, Rebecca Diya \\ Samuel ${ }^{1,2}$, Priyanka Arunachalam ${ }^{1}$, Chaluvanarayana H C $^{2}$, Pavan Belahalli ${ }^{2}$, Kalpana S R ${ }^{3}$, \\ Siddharth Jhunjhunwala ${ }^{1^{*}}$
}

1 - Centre for BioSystems Science and Engineering, Indian Institute of Science, Bengaluru, Karnataka, India - 560012

2 - Karnataka Institute of Endocrinology Research, Bengaluru, Karnataka, India - 560069

3 - Sri Jayadeva Institute of Cardiovascular Sciences and Research, Bengaluru, Karnataka, India 560069

* - address correspondence to Siddharth Jhunjhunwala (siddharth@iisc.ac.in), Centre for BioSystems Science and Engineering, Indian Institute of Science, Bengaluru, Karnataka, India 560012 


\begin{abstract}
Objective - The goal of this study was to identify biochemical and immunological parameters from the blood as predictors of non-healing in early-stage diabetic foot ulcers.
\end{abstract}

Research Design and Methods - We performed a cross-sectional prospective cohort study among individuals with early-stage foot ulcers visiting the Karnataka Institute of Endocrinology Research over a 2.5-year period. Histopathological, biochemical, and immunological data (a total of 31 parameters) from 52 individuals were collected and analyzed to determine if predictors of nonhealing may be identified. Data analysis was performed using traditional univariate analyses as well as univariate and multivariable logistic regression.

Results - Individual histopathological and biochemical parameters did not show any differences between healed and non-healed individuals. However, conventional univariate analysis and univariate logistic regression analysis showed that the expression of the cell-surface proteins CD63, HLA-DR and CD11b on monocytes $\left(\mathrm{CD} 14^{+}\right)$was significantly lower in non-healed individuals, but with moderate discriminative ability as assessed by area under the curve (AUC) of Receiver Operating Characteristics (ROC) curve. In comparison, a multivariable logistic regression model identified four of the 31 parameters to be salient predictors and demonstrated high discrimination ability with an AUC of ROC value of 0.87 . Among the four identified parameters, LDL cholesterol (OR 18.83, CI 18.83-342) and cell-surface expression of CD63 on monocytes (OR 0.12, CI 0.12-0.45) were significant. 
medRxiv preprint doi: https://doi.org/10.1101/2021.11.09.21266108; this version posted November 9, 2021. The copyright holder for this preprint (which was not certified by peer review) is the author/funder, who has granted medRxiv a license to display the preprint in perpetuity.

It is made available under a CC-BY 4.0 International license.

Conclusion - Through this study we conclude that LDL cholesterol and cell-surface expression of CD63 on monocytes are strong positive and negative predictors of non-healing, respectively, in individuals with early-stage DFU. Following validation in a larger cohort, these parameters may be used by the clinician for early identification of non-healers. 


\section{INTRODUCTION}

2

Diabetic foot ulcers (DFU) are chronic wounds that exhibit delayed healing in diabetic individuals. About 19-34\% of diabetic individuals are reported to be at risk of development of DFU(1). Currently, these wounds are managed through debridement, dressing, pressure off-loading, glycemic control and patient education(2). Treatment strategies include topical application of antibiotics and antimycotics to prevent infection, growth factor ointments to enhance cell proliferation for wound healing and negative pressure therapy for wound fluid drainage(2-4). Although a large proportion of early-stage wounds heal with timely care, a small but significant proportion of wounds fail to heal resulting in progression to gangrene formation necessitating limb amputation $(3,5,6)$. Hence, an early-stage predictor for wounds that are unlikely to heal would help the clinician alter treatment strategies, which might reduce the chance of amputation.

Measurable DFU characteristics such as wound size and depth have been described as potential predictors of non-healing in later stage and amputated wounds(7-9). In addition, it has been suggested that decreased wound area at early times (such as 1 and 4 weeks) correlated with poor healing at later time points (12 weeks)(8). However, precise cutoffs for wound size or depth, and wound area reduction are difficult to establish as it may vary between study cohorts, ulcer stages and ulcer types $(8,9)$. Chronic local inflammation (at the wound site) has also been correlated to delayed healing $(3,10)$. Some of the parameters associated with inflammation in DFUs include excessive myeloid cell infiltration(11), increased levels of pro-inflammatory mediators(12), presence of neutrophil extracellular traps (NETs)(13,14), and increased levels of MMP9 (15). However, quantification of such ulcer parameters is often difficult in many clinical centers. 
24 A few studies also suggest presence of chronic low-grade systemic inflammation in addition to 25 local inflammation in DFU individuals(16,17), quantification of which are possible in most clinics.

26 One such cohort study by Dinh and colleagues showed that non-healed individuals had increased

27 (as compared to individuals whose ulcers healed) serum levels of tumor necrosis factor- $\alpha$ (TNF-

$28 \alpha$, monocyte chemoattractant protein-1 (MCP-1), matrix metallopeptidase-9 (MMP-9), and

29 fibroblast growth factor-2 (FGF-2) about 8 months prior to the development of ulcers(18). We

30 conjectured that as myeloid cells play a vital role in the secretion of these cytokines and the

31 establishment of inflammation, they might contribute to slower or absence of healing. However,

32 their role in the healing of early-stage ulcers remains poorly characterized. The goal of the current

33 study was to characterize the phenotype of myeloid cells in individuals with active early-stage

34 ulcers and determine if their phenotype along with other clinical parameters may be used to predict 35 non-healing of ulcers. 


\section{METHODS}

Ethics statement

39 The study received approval from the institutional review board at the Karnataka Institute of Endocrinology Research (protocol number: IEC-KIER/04/28.10.2017). All procedures were

41 conducted in accordance with the approved protocol.

\section{Recruitment, Sample Collection, and Follow-Up}

44 Blood and wound biopsy samples were collected from individuals after obtaining informed

45 consent at Karnataka Institute of Endocrinology and Research (KIER), Bengaluru, Karnataka,

46 India. Stage of ulcer was graded by the clinician following Wagner's grade of ulcer

47 classification(19). Diabetic neuropathic patients who presented with a non-ischemic and

48 uninfected early-stage (stage I and II) foot ulcer were selected for the study (age range $40-70$

49 years). Patients presenting any associated complications such as significant cardiac or renal

50 ailments were excluded from the study. A total of 83 patients meeting patient inclusion criteria

51 provided informed consent and were recruited into the study. Among these, 31 individuals dropped

52 out from the study primarily because they did not show up for follow-up visits. Data from 52

53 individuals, for whom follow-up was performed twice a week for at least one month, were used

54 for further analysis.

55

56 Wound biopsy sample was collected before the debridement procedure, on the day of recruitment

57 right before initiation of treatment. Biopsy samples were stored in $10 \%$ buffered formalin until

58 further processing. Peripheral venous blood $(10 \mathrm{ml})$ was collected from enrolled patients within 7 
59 days of biopsy collection and used for biochemical testing and immunophenotyping. Patient's

60 wounds were dressed regularly. Wound healing was monitored for a period of one month by

61 capturing images using a near infra-red based camera (WoundZoom, USA) at the time of dressing.

62

Image analysis

64 Wound images captured by a near infra-red based camera were analyzed using ImageJ. Percentage

65 reduction in wound area was calculated as a measure of healing. Threshold of $50 \%$ reduction in

66 wound area on Day 30 was used to classify patients as healed.

67

Histopathological analysis

69 Grossing was performed on biopsy samples stored in formalin prior to processing to identify site

70 of ulcer and size. Dissected ulcerated tissue was then embedded in paraffin and sectioned. H\&E

71 stained slides were evaluated by a pathologist, who was blinded to the stage and healing status of

72 the patients. The pathologist assessed epidermal and vascular anomalies, inflammatory cell

73 infiltration, granulation tissue, collagen, and fibrin deposition.

74

\section{Biochemical parameter measurements}

76 Biochemical measurements were performed immediately following blood collection at the clinical

77 laboratory in KIER. Tests included blood glucose profile, complete hemogram, total lipid profile,

78 urine profile, thyroid profile, total liver function test, and few other additional parameters such as

79 serum creatinine, serum electrolytes and C-reactive protein. Salient biochemical parameters of

80 interest were chosen for further analysis.

81 


\section{Immunophenotyping}

83 Peripheral venous blood $(5 \mathrm{ml})$ was transported to the laboratories at Indian Institute of Science (IISc), where it was processed for further analysis (within 3 hours of blood collection to ensure analysis of granulocyte populations). Blood was centrifuged at $500 \mathrm{RCF}$ for 5 minutes to separate plasma from cells. Plasma was stored in $-80{ }^{\circ} \mathrm{C}$, while the pellet was subjected to RBC lysis using Ammonium-Chloride-Potassium (ACK) lysis buffer at 1:12.5 (blood to lysis buffer) ratio by volume. Lysis was performed at room temperature (RT) for 10 minutes and quenched using three times the volume using $1 \mathrm{X}$ PBS buffer containing 4mM EDTA. Samples were centrifuged at 400 RCF for 4 minutes at $4{ }^{\circ} \mathrm{C}$. Supernatant was decanted and pellet was re-suspended in $1 \mathrm{~mL}$ of $1 \mathrm{X}$ PBS EDTA buffer. An aliquot of the sample was stained with BD Horizon ${ }^{\mathrm{TM}}$ Fixable Viability Stain 510 to stain dead cells at a concentration of $0.3 \mu 1 / 100 \mu 1$ volume of 1 million cell suspension. Samples were incubated in dark at RT for 20 minutes before quenching with $1 \mathrm{~mL}$ of $1 \mathrm{X}$ PBS containing $1 \% \mathrm{BSA}$ and $4 \mathrm{mM}$ EDTA (staining buffer). Cells were then fixed using $2 \%$ paraformaldehyde for 30 minutes at $4{ }^{\circ} \mathrm{C}$. Samples were washed and re-suspended in $1 \mathrm{~mL}$ staining buffer for staining with fluorophore conjugated antibodies procured from BD Biosciences.

Staining was performed according to manufacturer's recommended dilutions. Antibodies used are summarized in Supplementary Table 1. Samples were quenched with $1 \mathrm{~mL}$ staining buffer following staining, washed, and re-suspended in $300 \mu \mathrm{l}$ buffer for acquisition on flow cytometer (BD FACSCelesta ${ }^{\mathrm{TM}}$ Cell Analyzer, BD Biosciences, USA). Appropriate single-color controls were prepared using compensation beads (BD Biosciences, USA), which were stained with antibodies following manufacturer's protocol. Additionally, fluorescence minus one (FMO) controls were prepared to correct for group effect of fluorophores on spectral spill. Briefly, live- 
dead dye stained cells were stained with antibodies for all colors except one which was replaced by respective isotype to prepare FMO for a color of interest. Similar controls were prepared for all fluorophores mentioned in Supplementary Table 1 and used for intensity correction at the time of data analysis. Voltages were set on system using compensation beads at the time of sample acquisition and a minimum of $100,000 \mathrm{CD}^{4} 5^{+}$live events were acquired using BD FACSDiva ${ }^{\mathrm{TM}}$ Version 6 Software system (BD Biosciences, USA).

\section{Flow cytometry data analyses}

All data analyses were performed on FCS files using FlowJo ${ }^{\mathrm{TM}}$ v10.6 (Becton Dickinson, USA). Compensation was performed on FlowJo using compensation beads. CD14 and CD15 were used to identify monocytes and granulocytes, respectively. FMO's were used to draw appropriate gates. A threshold of minimum 100 events was set to report percentage positive cells and median fluorescence intensity (MFI) values for all markers expressed by $\mathrm{CD} 14^{+}$and $\mathrm{CD} 15^{+}$cells. Corresponding FMO values of markers were subtracted to correct for group effect of fluorophores on spectral spill. Corrected MFI values were tabulated for further analyses.

\section{Data preprocessing and logistic regression model}

Biochemical and immunological data of all patients was combined into one matrix and standardized prior analysis. Standardization was performed by centering data around mean and dividing each observation by standard deviation. Univariate and multivariable logistic regression models were built using standardized data using $g l m$ function in stats package of R. Stepwise feature selection was performed using packages $M A S S(20)$ and magrittr(21) with low Akaike's information criterion (AIC) score as selection criterion for identifying salient predictors. 
128 Unadjusted odd's ratio was reported with 95\% confidence interval for multivariable logistic

129 regression model. ROC analysis was performed using $p R O C$ package in $\mathrm{R}(22)$. ROC plots were

130 created using the same package for univariate and multivariable logistic regression models.

\section{Statistics}

133 Fisher's exact test was performed to analyze categorical histology data in R using stats package. 134 Heatmap was created using ComplexHeatmap package in $\mathrm{R}(23)$. Univariate analysis of all 135 predictors was performed using student's t-test with Welch's correction using GraphPad Prism 8. 136 Wald's test for significance of predictors was performed by glmnet package as part of the routine.

137 Model evaluation statistics such as Chi-square goodness of fit (GOF) test and Hosmer-Lemeshow 138 (HL) test was performed using stats and ResourceSelection (24) package in R respectively. AIC 139 score was obtained for all models using stats package. Model fit plots were created using ggplot2 140 package in $\mathrm{R}(25)$. RStudio 4.1.0 was used for all $\mathrm{R}$ based analyses. Raw data and R scripts used 141 for performing analyses are uploaded

on 


\section{RESULTS}

\section{Cohort characteristics}

146 The final study cohort comprised of 52 diabetic individuals, with either Stage I (30) or Stage II

147 (22) foot ulcers. Their clinical characteristics are summarized in Table 1. We observe an

148 underrepresentation of females in the study (only 8 females in comparison to 44 males), which 149 could be due to sociological reasons. Through regular follow-up for one month that involved 150 standard clinical care and imaging of the wound (Supplementary Figure 1), we determined that the ulcers had healed ( $>50 \%$ reduction in wound area) in 33 individuals, while it remained non-healed

152 in 19 individuals. Our healing data shows that ulcers healed in $66.67 \%$ of stage I and $59 \%$ of stage

153 II foot ulcer individuals with the standard care provided at our clinic.

\section{Histopathological analysis of wound biopsy}

156 Hematoxylin and Eosin (HE) stained sections of the ulcer biopsies were analyzed by a pathologist

157 to assess the damage to skin tissue and levels of inflammatory cell infiltration. The pathologists 158 report revealed that while presence of granulation tissue correlated with stage of ulcer, there was 159 an absence of correlation between any of the histological features and healing of ulcers 160 (Supplementary Figure 2A and 2B). This suggests that histopathological parameters may not be good predictors of ulcer healing.

\section{Univariate analysis of biochemical and immunological parameters}

164 We also collected peripheral venous blood from the recruited individuals for biochemical analysis 165 and immunological characterization of neutrophils and monocytes in circulation. Immunological 
168 (Supplementary Figure 3B).

The standardized numerical values of the data collected from both biochemical analysis and

171 immunological characterization (parameters) are presented as a heat-map in Figure 1. A clear

172 separation among parameters was not observed between healed and non-healed individuals.

173 Nevertheless, a univariate analysis was performed using Student's t-test with Welch's correction

174 on each parameter, and this analysis revealed that none of the biochemical parameters showed

175 significant differences among the healed and non-healed individuals (Supplementary Table 2).

176 However, the analysis did show that the expression levels of three proteins on monocytes (CD14

177 expressing cells), CD63, HLA-DR and CD11b, were significantly higher on individuals whose

178 ulcers had healed as compared to those whose ulcers had not healed (Supplementary Table 2 and

179 Supplementary Figure 4). When a receiver operator curve (ROC) analysis was performed

180 (Supplementary Table 3) to assess the discriminative capability of each parameter, all three were

181 observed to have relatively moderate area under the curve (AUC) values and were deemed to be

182 not effective in discriminating between non-healed ulcers and healed ulcers. Hence, we next used

183 logistic regression, a binary classifier model to assess outcome prediction.

185 Univariate logistic regression analysis

186 A univariate logistic regression analysis was performed to evaluate predictive capability of the

187 three individual parameters identified above. In this analysis, the parameters MFI of CD63 and

188 HLA-DR among monocytes $\left(\mathrm{CD}^{+} 4^{+}\right)$were observed to be significant according to the Chi-square 
Goodness of Fit (GOF) test, and only MFI of CD63 among monocytes (CD14 ${ }^{+}$) was significant when evaluated using the Wald's test (Supplementary Table 4). Additionally, the odd's ratio (OR) for MFI of CD63 among monocytes $\left(\mathrm{CD}^{+} 4^{+}\right)$was found to be 0.26 (CI 0.08-0.68) suggesting strong negative association with healing outcome. The univariate logistic regression model's prediction against actual outcomes for each univariate model are shown in Figure 2A. Further, ROC analysis was performed to evaluate the discriminative capability of univariate logistic regression models (Figure 2B). AUC was found to be 0.73 for CD63, 0.65 for HLA-DR and 0.63 for $\mathrm{CD} 11 \mathrm{~b}$ expressing monocytes indicating that the discriminative ability of the univariate logistic regression models was not better than the traditional univariate analysis. Hence, we next performed a multivariable logistic regression analysis.

\section{Multivariable logistic regression analysis}

201 To determine if a combination of parameters enhances model performance, two multivariable logistic regression models were built initially. One model included only the biochemical and cellular parameters while the other included only immunological phenotypic parameters. Stepwise

204 feature selection was performed to identify and retain only salient predictors to build the model. six salient parameters were identified by stepwise feature selection technique, four of which were

207 significant according to Wald's test (Table 2). The model performance was found to be significant 208 based on a Chi-square goodness of fit $(\mathrm{GOF})$ test $(\mathrm{p}=0.011)$. A Hosmer-Lemeshow $(\mathrm{HL})$ goodness 209 of fit test was also performed to evaluate agreement between model's predicted and expected event rates across deciles of risk groups. HL test $\mathrm{p}$ value was found to be $0.411 \mathrm{implying}$ lack of evidence

211 for disagreement between predicted and expected event rates. Additionally, Akaike Information 
212 Criterion (AIC), an estimator of out-of-sample prediction error to assess model performance was

213 found to be 65.74. Odds ratio for each predictor along with CI is summarized in Table 2, and

214 Figure 3A shows the predicted probabilities of the model as well as the ROC analysis.

216 Similarly, a stepwise feature selection identified four of 15 immunological phenotypic parameters

217 to build the Immunological model, three of which were found to be significant according to Wald's

218 test (Table 3). Chi-square and HL GOF tests showed p value 0.002 and 0.368 , respectively

219 indicating that the model is statistically significant, and the AIC was found to be 61.21. Odd's ratio

220 and corresponding CI are summarized in Table 3. Figure 3B shows the predicted probabilities of

221 the model as well as the ROC analysis.

223 While both the biochemical and immunological models of multivariable logistic regression

224 showed that specific groups of parameters could help to predict non-healing, we were interested

225 in determining if combining the parameters would enhance model performance. For such an

226 analysis, significant parameters of both the multivariable models were chosen and a stepwise

227 feature selection was performed to retain only salient parameters in the model. This new model,

228 with four parameters, showed an excellent fit (Figure 4A), an improved AUC of 0.87 in the ROC

229 analysis (Figure 4B), and a lowered AIC score of 56.25. The Chi-square and HL GOF test show $p$

230 value 0.0002 and 0.3086 , respectively indicating that the model is statistically significant. Two

231 (LDL cholesterol and CD63 MFI among monocytes) of the four selected parameters showed

232 significance as assessed by Wald's test (Table 4). LDL cholesterol showed an OR 18.83 (CI 18.83-

233 342) indicating positive association with healing outcome, while the MFI of CD63 among

234 monocytes $\left(\mathrm{CD}^{+} 4^{+}\right)$showed an OR 0.12 (CI 0.02-0.45) suggesting a negative association with 
medRxiv preprint doi: https://doi.org/10.1101/2021.11.09.21266108; this version posted November 9, 2021. The copyright holder for this preprint (which was not certified by peer review) is the author/funder, who has granted medRxiv a license to display the preprint in perpetuity.

It is made available under a CC-BY 4.0 International license .

235 healing outcome. Together, these metrics suggest that the combined model performed the best in

236 predicting non-healing among individuals with early-stage diabetic foot ulcers. 


\section{DISCUSSION}

239 The goal of our study was to identify clinically measurable parameters that could help predict

240 individuals in whom early-stage DFUs fail to heal. Hence, we focused on collecting and analyzing

241 histopathological, biochemical, and immunological parameters that are relatively easy to collect

242 in many health-centers. Our analysis revealed that the histopathological data did not correlate with

243 healing, and no individual biochemical or immunological parameter was a good predictor of non-

244 healing. However, multivariable logistic regression analysis using a combination of biochemical

245 and immunological parameters was able to predict non-healing. Specifically, the combined

246 analysis determined that serum LDL cholesterol (OR 18.83, CI 2.22 and 342.00) and the

247 expression level of the protein CD63 among monocytes (OR 0.12, CI 0.02 and 0.45) were

248 significant predictors of non-healing in early-stage DFUs.

250 Previous cohort studies on individuals with DFU have identified systemic inflammatory mediators

251 that appear to correlate with inflammation at the wound site and non-healing. For example, Veves

252 and colleagues report the presence of increased serum levels of TNF- $\alpha$, FGF-2, MCP-1 and MMP-

2539 in individuals who developed DFU eight months after the serum measurements and failed to heal

254 by 12 weeks(18). Recently, the same authors identified unique transcriptomic signatures in

255 individuals with foot ulcers and those that do not heal(26). Other studies also show that neutrophil

256 elastase and citrullinated H3, markers of neutrophils extracellular traps (NETs), were observed to

257 be increased in plasma of non-healed DFU individuals compared to healed individuals(14,27). In

258 terms of diabetic wound-specific biomarkers for healing, independent studies by Wong et al,

259 Fadini et al and Yang et al observed an increased presence of NET components in wounds of 
$261(13,14,27)$. While these studies clearly establish that both local and systemic inflammation

262 correlate with poor healing, measurement of the identified mediators are often difficult due to the

263 labor-intensive methodologies involved or the requirement for equipment that are not yet available

264 in many clinical centers.

266 Facile measurable parameters such as wound characteristics (wound size and area) have also been

267 reported as predictors of non-healing. Sheehan et al. showed that the percentage change in wound

268 area after four weeks was a robust predictor of non-healing observed at twelve weeks(7). Further,

269 Lavery et al. demonstrated that percentage reduction in wound area by one week was a strong

270 predictor for non-healing at sixteen weeks(8). However, these observations were from patients

271 with late stage or amputated wounds, which may not be applicable to early-stage wounds.

272 Additionally, precise cutoffs of wound dimension changes are also difficult to establish, as they

273 may vary between study cohorts, ulcer type, and stage of wounds. In this context, serum

274 biochemical indicators that are routinely measured in the clinic and immunological phenotyping

275 (which is becoming common in many tertiary care centers) are easy-to-use as potential predictors

276 of wound healing.

277

278 The major strength of our study is the identification of such measurable biochemical and

279 immunological parameters as predictors of non-healing in early-stage DFU individuals. Our study

280 shows that LDL cholesterol among biochemical parameters and expression level of protein CD63

281 on monocytes among immunological parameters (along with total cholesterol and percentage of

282 monocytes), together, serve as strong predictors of non-healing in individuals with active foot

ulcers. LDL cholesterol has been implicated in inflammation and dysregulated innate immune cell 
284 function(28). Hence, individuals with increased levels of LDL cholesterol may have systemic low285 grade inflammation, which might impact many tissues, including DFUs. Additionally, LDL cholesterol has been shown to have inhibitory effect on endothelial cell proliferation, and has been

287 shown to cause delays in wound healing in mouse models of research(29). In concurrence with these observations, our analysis reveals that higher levels of LDL cholesterol correlate with nonhealing of early-stage foot ulcers, possibly through the establishment of higher systemicinflammatory levels and lowered endothelial cell proliferation.

The protein CD63 is known to be an important component of phagosomes(30) and plays a role in trafficking of proteins from the membrane into the cell (31). Additionally, upon maturation of monocytes, surface expression of CD63 is known to decrease(32). Monocytes in circulation are known to be recruited to the wound bed where they differentiate into macrophages releasing pro and anti-inflammatory mediators to accelerate healing(33). While the exact link between CD63 and monocyte activity in wound healing (or microbial killing) is not clear, we speculate that a circulating monocyte with lower surface CD63 expression might indicate matured cell with altered phenotype or a cell with reduced ability to engage in wound healing. Such a state could explain the inverse relationship between monocytic CD63 expression and healing of diabetic wounds. inflammation (34-36). In that context, a surprising observation in our study is the lack of correlation between hyperglycemia (both HbA1c and fasting blood sugar) and non-healing. This observation is in contrast to a decade old retrospective study by Christman et al., which showed that higher HbA1c strongly correlates to poor healing in DFU individuals(37). Two possible 
reasons for the difference between our observations and this study, is the stage of ulcer under consideration (we focus on early-stage ulcers compared to aggressive late stage or amputated wounds in the other study) and the method used to classify healing (we measure healing at an endpoint compared to rate of healing used in the other study). However, our data and observations

311 are in line with a meta-analysis performed on individuals with neuropathic diabetic wounds(38)

312 and a more recent retrospective study on individuals with DFU (39). Based on these studies and 313 our observations, it does appear that while hyperglycemia may play a role in the development of

314 foot ulcers, it does not correlate with healing status.

One of the major limitations of this study is the relatively small sample size of the cohort, which

317 may limit the statistical power. Nevertheless, our observations provide new information on measurable predictors for non-healing. Second is the classification system used for assessing 319 healing status, which was set as 50\% reduction in wound area by 4 weeks following thresholds 320 reported by Sheehan et al. and Lavery et al. $(7,8)$. Due to challenges associated with following-up 321 patients in our setting, we were unable to physically verify if the individuals classified as healed 322 showed complete wound healing by $\sim 16$ weeks. Third, the duration that the individual has had an 323 ulcer for before presentation in the clinic was determined through an oral conversation but could 324 not be verified as in many cases no prior medical records exist. Lastly, many of the individuals 325 who visit this center have uncontrolled diabetes, and while this may be common for many 326 government health centers in India, it may not be the norm elsewhere. 
In summary, our cross-sectional cohort study analyzing clinical, biochemical, and immunological parameters among individuals with early-stage DFU showed that no single parameter correlates effectively with non-healing of wounds. However, a multivariable logistic regression model of the data suggests that LDL cholesterol and the expression level of CD63 on monocytes are strong and significant predictors of non-healing, when used along with total cholesterol and percentage of monocytes. While validation studies in larger cohorts are required, our observations have the potential to aid clinicians in identifying individuals at the risk of a poor healing outcome, and hence pursue more aggressive or alternate treatment strategies.

\section{ACKNOWLEDGMENTS}

339 The authors acknowledge support from the Rajiv Gandhi University of Health Sciences, Govt. of Karnataka (17C008B). This study was also funded in part by the Biodesign and Bioengineering

341 Initiative (Phase II), Department of Biotechnology, Govt. of India. It was also funded by the Dr.

342 Vijaya and Rajagopal Rao laboratory for Biomedical Engineering at IISc. The authors have no conflict of interest to declare. JVR helped design the study, collected the immunological data, analyzed all data, and wrote the manuscript. SK and PA assisted with immunological data collection. VKD and RDS helped with subject recruitment and assisted with biopsy as well as

346 immunological sample processing. CHC and PB assisted with study design, recruited subjects, 347 collected the biopsies, and assisted with writing the manuscript. KSR helped with study design, 348 performed the histopathological analysis and assisted with writing the manuscript. SJ designed the study, assisted with data collection and analysis, and wrote the manuscript. 


\section{REFERENCES}

1. Armstrong DG, Boulton AJM, Bus SA. Diabetic Foot Ulcers and Their Recurrence [Internet]. http://dx.doi.org/10.1056/NEJMra1615439. Massachusetts Medical Society; 2017 [cited 2021 Jun 10]. Available from:

https://www.nejm.org/doi/10.1056/NEJMra1615439

2. Everett E, Mathioudakis N. Update on management of diabetic foot ulcers. Ann N Y Acad Sci. 2018 Jan;1411(1):153-65.

3. Baltzis D, Eleftheriadou I, Veves A. Pathogenesis and Treatment of Impaired Wound Healing in Diabetes Mellitus: New Insights. Adv Ther. 2014 Aug;31(8):817-36.

4. Laiva AL, O'Brien FJ, Keogh MB. Innovations in gene and growth factor delivery systems for diabetic wound healing. J Tissue Eng Regen Med. 2018 Jan;12(1):e296-312.

5. Jeffcoate WJ, Chipchase SY, Ince P, Game FL. Assessing the outcome of the management of diabetic foot ulcers using ulcer-related and person-related measures. Diabetes Care. 2006 Aug;29(8):1784-7.

6. Boulton AJM. The pathway to foot ulceration in diabetes. Med Clin North Am. 2013 Sep;97(5):775-90.

7. Sheehan P, Jones P, Caselli A, Giurini JM, Veves A. Percent Change in Wound Area of Diabetic Foot Ulcers Over a 4-Week Period Is a Robust Predictor of Complete Healing in a 12-Week Prospective Trial. Diabetes Care. 2003 Jun 1;26(6):1879-82.

8. Lavery LA, Barnes SA, Keith MS, Seaman JW, Armstrong DG. Prediction of Healing for Postoperative Diabetic Foot Wounds Based on Early Wound Area Progression. Diabetes Care. 2008 Jan 1;31(1):26-9.

9. Vella L, Formosa C. Characteristics Predicting the Outcome in Individuals with Diabetic Foot Ulcerations. Journal of the American Podiatric Medical Association. 2017 May 1;107(3):180-91.

10. Acosta JB, Garcia del Barco D, Cibrian Vera D, Savigne W, Lopez-Saura P, Guillen Nieto $\mathrm{G}$, et al. The pro-inflammatory environment in recalcitrant diabetic foot wounds. International Wound Journal. 2008 Oct;5(4):530-9.

11. Joshi N, Pohlmeier L, Ben-Yehuda Greenwald M, Haertel E, Hiebert P, Kopf M, et al. Comprehensive characterization of myeloid cells during wound healing in healthy and healing-impaired diabetic mice. Eur J Immunol. 2020 Sep;50(9):1335-49.

12. Ochoa O, Torres FM, Shireman PK. Chemokines and Diabetic Wound Healing. Vascular. 2007 Dec 1;15(6):350-5.

13. Wong SL, Demers M, Martinod K, Gallant M, Wang Y, Goldfine AB, et al. Diabetes primes neutrophils to undergo NETosis, which impairs wound healing. Nat Med. 2015 Jul;21(7):815-9.

14. Fadini GP, Menegazzo L, Rigato M, Scattolini V, Poncina N, Bruttocao A, et al. NETosis Delays Diabetic Wound Healing in Mice and Humans. Diabetes. 2016 Apr;65(4):1061-71.

15. Martins VL, Caley M, O’Toole EA. Matrix metalloproteinases and epidermal wound repair. Cell Tissue Res. 2013 Feb;351(2):255-68.

16. Williams MD, Nadler JL. Inflammatory mechanisms of diabetic complications. Curr Diab Rep. 2007 Jun 1;7(3):242-8.

17. Pickup JC, Crook MA. Is Type II diabetes mellitus a disease of the innate immune system? Diabetologia. 1998 Sep 18;41(10):1241-8. 
18. Dinh T, Tecilazich F, Kafanas A, Doupis J, Gnardellis C, Leal E, et al. Mechanisms Involved in the Development and Healing of Diabetic Foot Ulceration. Diabetes. 2012 Nov $1 ; 61(11): 2937-47$.

19. Wagner FW. The dysvascular foot: a system for diagnosis and treatment. Foot Ankle. 1981 Sep;2(2):64-122.

20. Venables WN, Ripley BD. Modern Applied Statistics with S [Internet]. 4th ed. New York: Springer-Verlag; 2002 [cited 2021 Sep 29]. (Statistics and Computing). Available from: https://www.springer.com/gp/book/9780387954578

21. magrittr) SMB (Original author and creator of, Wickham H, Henry L, RStudio. magrittr: A Forward-Pipe Operator for R [Internet]. 2020 [cited 2021 Sep 21]. Available from: https://CRAN.R-project.org/package=magrittr

22. Robin X, Turck N, Hainard A, Tiberti N, Lisacek F, Sanchez J-C, et al. pROC: an opensource package for R and $\mathrm{S}+$ to analyze and compare ROC curves. BMC Bioinformatics. 2011 Mar 17;12(1):77.

23. Gu Z, Eils R, Schlesner M. Complex heatmaps reveal patterns and correlations in multidimensional genomic data. Bioinformatics. 2016 Sep 15;32(18):2847-9.

24. Sólymos P, Lele SR. Revisiting resource selection probability functions and single-visit methods: clarification and extensions. Methods in Ecology and Evolution. 2016;7(2):196205.

25. Wickham H. ggplot2: Elegant Graphics for Data Analysis [Internet]. 2nd ed. Springer International Publishing; 2016 [cited 2021 Sep 21]. (Use R!). Available from: https://www.springer.com/gp/book/9783319242750

26. Theocharidis G, Thomas BE, Sarkar D, Pilcher WJ, Dwivedi B, Sandoval-Schaefer T, et al. Single Cell Transcriptomic Landscape of Diabetic Foot Ulcers [Internet]. 2021 Mar [cited 2021 Oct 25] p. 2021.03.11.434413. Available from: https://www.biorxiv.org/content/10.1101/2021.03.11.434413v1

27. Yang S, Gu Z, Lu C, Zhang T, Guo X, Xue G, et al. Neutrophil Extracellular Traps Are Markers of Wound Healing Impairment in Patients with Diabetic Foot Ulcers Treated in a Multidisciplinary Setting. Adv Wound Care (New Rochelle). 2020 Jan 1;9(1):16-27.

28. Jukema RA, Ahmed TAN, Tardif J-C. Does low-density lipoprotein cholesterol induce inflammation? If so, does it matter? Current insights and future perspectives for novel therapies. BMC Medicine. 2019 Nov 1;17(1):197.

29. Bogachkov YY, Chen L, Le Master E, Fancher IS, Zhao Y, Aguilar V, et al. LDL induces cholesterol loading and inhibits endothelial proliferation and angiogenesis in Matrigels: correlation with impaired angiogenesis during wound healing. American Journal of Physiology-Cell Physiology. 2020 Apr 1;318(4):C762-76.

30. Artavanis-Tsakonas K, Love JC, Ploegh HL, Vyas JM. Recruitment of CD63 to Cryptococcus neoformans phagosomes requires acidification. PNAS. 2006 Oct 24;103(43):15945-50.

31. Pols MS, Klumperman J. Trafficking and function of the tetraspanin CD63. Experimental Cell Research. 2009 May 15;315(9):1584-92.

32. Tippett E, Cameron PU, Marsh M, Crowe SM. Characterization of tetraspanins CD9, CD53, CD63, and CD81 in monocytes and macrophages in HIV-1 infection. J Leukoc Biol. 2013 Jun;93(6):913-20. 
33. Krzyszczyk P, Schloss R, Palmer A, Berthiaume F. The Role of Macrophages in Acute and Chronic Wound Healing and Interventions to Promote Pro-wound Healing Phenotypes. Frontiers in Physiology. 2018;9:419.

34. Rekeneire N de, Peila R, Ding J, Colbert LH, Visser M, Shorr RI, et al. Diabetes, Hyperglycemia, and Inflammation in Older Individuals: The Health, Aging and Body Composition study. Diabetes Care. 2006 Aug 1;29(8):1902-8.

35. Tsalamandris S, Antonopoulos AS, Oikonomou E, Papamikroulis G-A, Vogiatzi G, Papaioannou S, et al. The Role of Inflammation in Diabetes: Current Concepts and Future Perspectives. Eur Cardiol. 2019 Apr;14(1):50-9.

36. Morey M, O'Gaora P, Pandit A, Hélary C. Hyperglycemia acts in synergy with hypoxia to maintain the pro-inflammatory phenotype of macrophages. PLOS ONE. 2019 Aug 15;14(8):e0220577.

37. Christman AL, Selvin E, Margolis DJ, Lazarus GS, Garza LA. Hemoglobin A1c Predicts Healing Rate in Diabetic Wounds. Journal of Investigative Dermatology. 2011 Oct 1;131(10):2121-7.

38. Margolis DJ, Kantor J, Santanna J, Strom BL, Berlin JA. Risk Factors for Delayed Healing of Neuropathic Diabetic Foot Ulcers: A Pooled Analysis. Archives of Dermatology. 2000 Dec 1;136(12):1531-5.

39. Fesseha BK, Abularrage CJ, Hines KF, Sherman R, Frost P, Langan S, et al. Association of Hemoglobin A1c and Wound Healing in Diabetic Foot Ulcers. Diabetes Care. 2018 Jul $1 ; 41(7): 1478-85$. 
TABLES

\begin{tabular}{|ccccc|}
\hline & Stage of ulcer & Median & LCI & UCI \\
\hline \multirow{2}{*}{ Age } & I & 52.50 & 49.47 & 55.53 \\
& II & 54.50 & 50.55 & 58.45 \\
\hline \multirow{2}{*}{ BMI } & I & 25.54 & 24.15 & 26.93 \\
& II & 27.26 & 25.67 & 28.84 \\
\hline \multirow{2}{*}{ HbA1c \% } & I & 9.35 & 8.72 & 9.98 \\
& II & 9.30 & 8.71 & 9.89 \\
\hline \multirow{2}{*}{ FBS (mg/dl) } & I & 155.50 & 140.02 & 170.98 \\
& II & 147.00 & 123.87 & 170.13 \\
\hline \multirow{2}{*}{ PPBS (mg/dl) } & I & 217.00 & 192.10 & 241.90 \\
& II & 221.00 & 186.06 & 255.94 \\
\hline
\end{tabular}

Table 1: Clinical and blood sugar characteristics of recruited individuals. Data from 30 individuals with stage I and 22 individuals with stage 2 ulcers is presented here. LCI and UCI indicate lower and upper confidence intervals, respectively. 


\begin{tabular}{|c|ccc|c|}
\hline \multicolumn{7}{|c}{ OR Statistics } \\
\hline Parameters & OR & LCI & UCI & Wald's test \\
\hline Total Cholesterol & $4.88 \mathrm{E}-03$ & $1.29 \mathrm{E}-05$ & 0.57 & $0.047^{*}$ \\
\hline LDL Cholesterol & 224.0706 & 3.78 & $4.34 \mathrm{E}+04$ & $0.021^{*}$ \\
\hline Triglycerides & $4.63 \mathrm{E}-27$ & $5.23 \mathrm{E}-63$ & $1.93 \mathrm{E}+05$ & 0.119 \\
\hline VLDL Cholesterol & $6.75 \mathrm{E}+26$ & $9.22 \mathrm{E}-06$ & $1.32 \mathrm{E}+63$ & 0.115 \\
\hline$\%$ CD $14^{+}$cells & 2.45 & 1.16 & 5.97 & $0.029 *$ \\
\hline$\%$ CD $15^{+}$cells & 2.42 & 1.09 & 6.51 & $0.046^{*}$ \\
\hline
\end{tabular}

Table 2: Summary of multivariable Biochemical logistic regression model. OR indicates

Odds ratio and LCI and UCI indicate lower and upper confidence interval, respectively. Wald's test was used to assess significance of predictors contributing to model's performance. * indicates significance with $\mathrm{p}<0.05$ 


\begin{tabular}{|c|c|c|c|c|}
\hline & \multicolumn{3}{|c|}{ OR Statistics } & p value \\
\hline Parameters & OR & LCI & UCI & Wald's test \\
\hline MFI of CD11b on CD15 & 3.23 & 0.64 & 23.00 & 0.177 \\
\hline MFI of CD63 on CD14 & 0.24 & 0.06 & 0.70 & $0.019 *$ \\
\hline MFI of CD282 on CD14 & 4.03 & 1.28 & 19.10 & $0.036^{*}$ \\
\hline MFI of CD11b on CD14 & 0.07 & 0.00 & 0.57 & $0.044^{*}$ \\
\hline
\end{tabular}

Table 3: Summary of multivariable Immunological logistic regression model. OR indicates

Odds ratio and LCI and UCI indicate lower and upper confidence interval, respectively. Wald's test was used to assess significance of predictors contributing to model's performance. * indicates significance with $\mathrm{p}<0.05$ 


\begin{tabular}{|c|ccc|c|}
\hline \multicolumn{5}{|c}{ OR Statistics } \\
\hline Parameters & OR & LCI & UCI & Wald's test \\
\hline Total Cholesterol & 0.15 & 0.01 & 1.07 & 0.105 \\
\hline LDL Cholesterol & 18.83 & 2.22 & 342.00 & $0.021^{*}$ \\
\hline$\%$ CD14 ${ }^{+}$cells & 2.18 & 1.03 & 5.40 & 0.057 \\
\hline MFI of CD63 on CD14 & 0.12 & 0.02 & 0.45 & $0.006^{* *}$ \\
\hline
\end{tabular}

Table 4: Summary of multivariable combined logistic regression model. OR indicates Odds ratio and LCI and UCI indicate lower and upper confidence interval, respectively. Wald's test was used to assess significance of predictors contributing to model's performance. * indicates significance with $\mathrm{p}<0.05 * *$ indicate $\mathrm{p}<0.01$ 
medRxiv preprint doi: https://doi.org/10.1101/2021.11.09.21266108; this version posted November 9, 2021. The copyright holder for this preprint (which was not certified by peer review) is the author/funder, who has granted medRxiv a license to display the preprint in perpetuity.

It is made available under a CC-BY 4.0 International license.

\section{FIGURES}

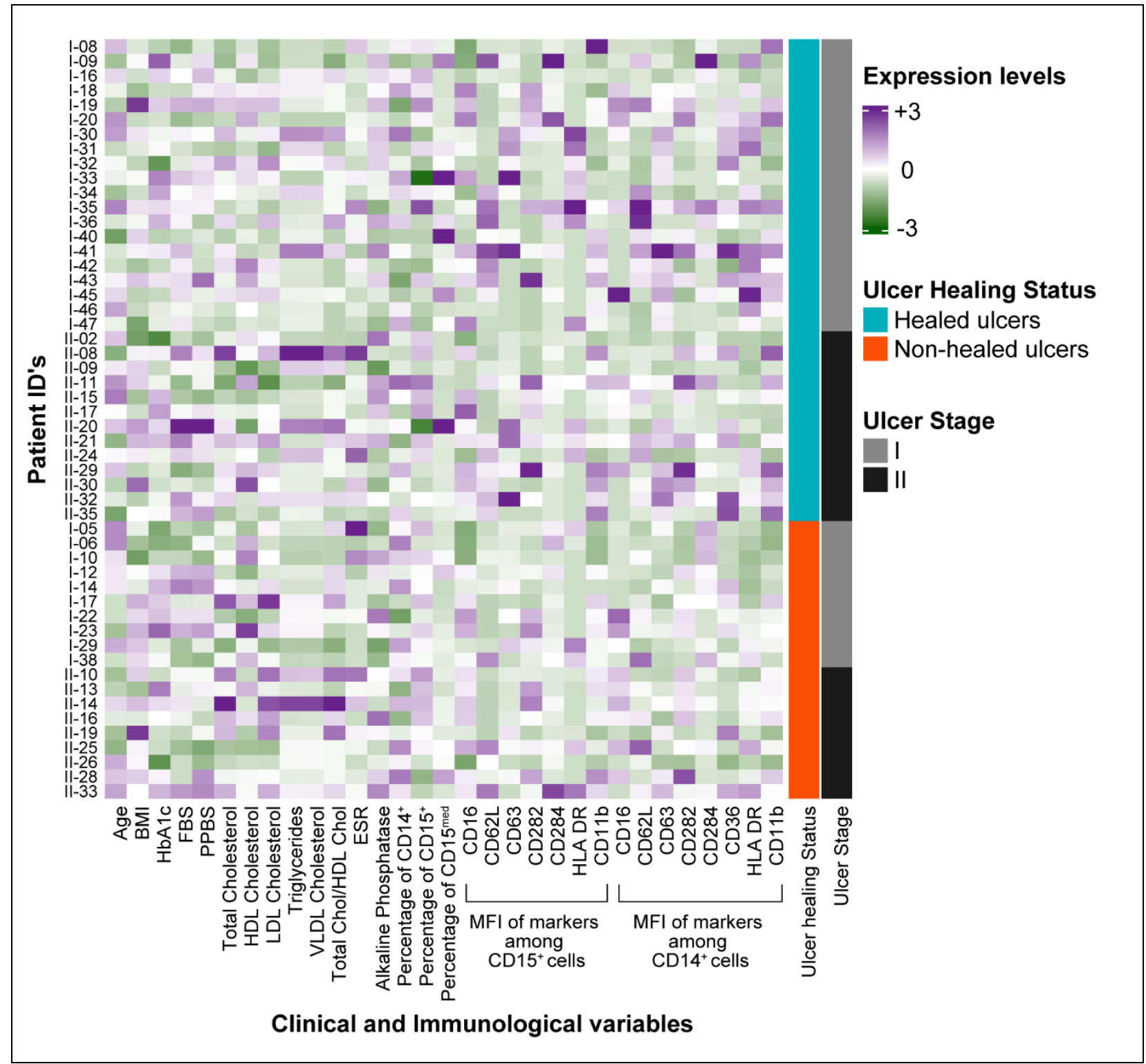

Figure 1: Heatmap of standardized biochemical and immunological parameters measured

in 52 individuals with diabetic foot ulcer. Standardized data of each parameter is represented as

heatmap with intensities varying between brown (-3), white (0) and purple (+3). Annotation Ulcer healing status indicates if the individual was classified as healed or non-healed after 1 month follow-up, and Ulcer stage represents the stage of ulcer in each individual. 


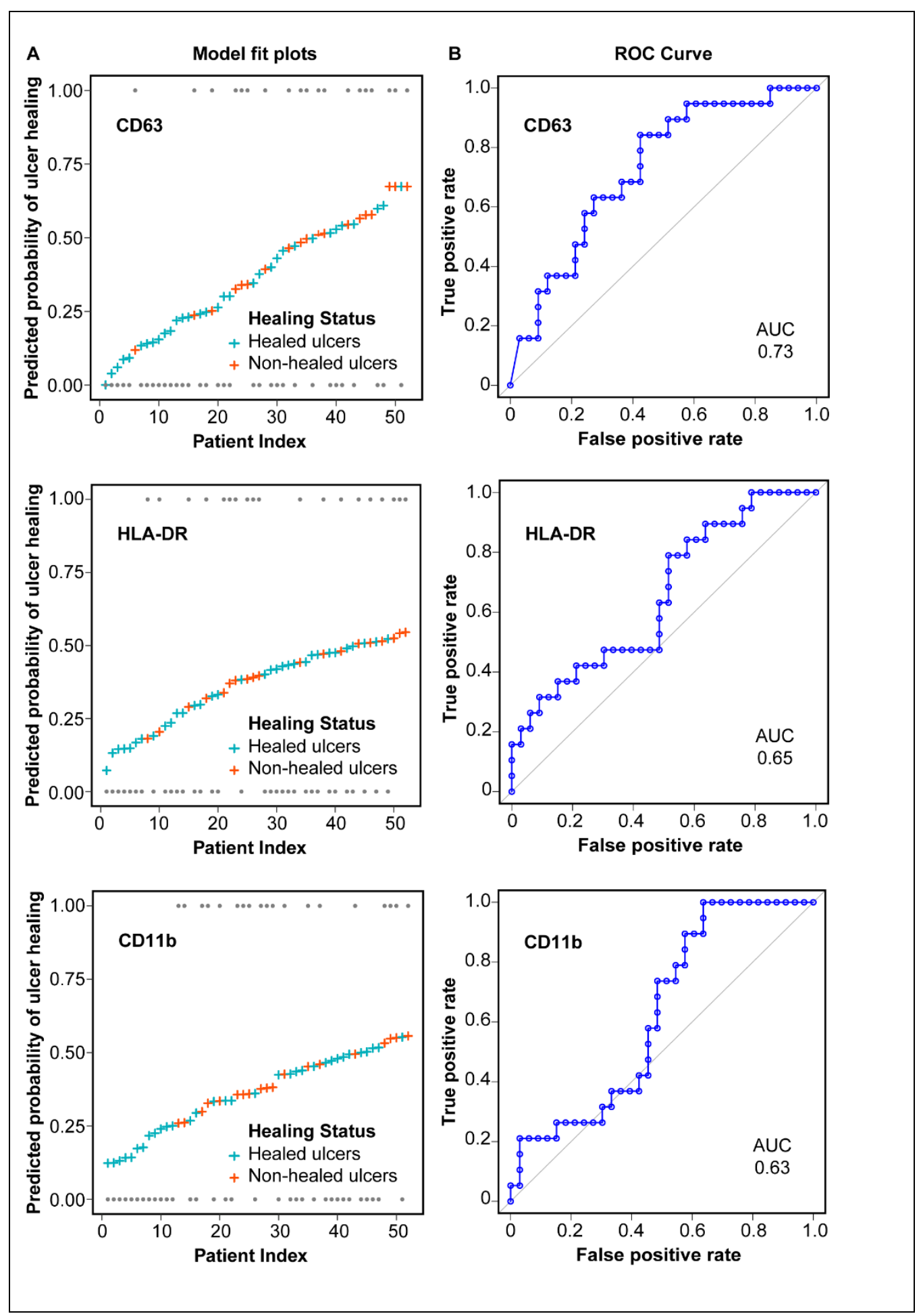

Figure 2: Univariate logistic regression model. A - The predicted probabilities CD63, HLA-DR and CD11b are shown (blue - healed; orange - non-healed) against reference probabilities 
medRxiv preprint doi: https://doi.org/10.1101/2021.11.09.21266108; this version posted November 9, 2021. The copyright holder for this preprint (which was not certified by peer review) is the author/funder, who has granted medRxiv a license to display the preprint in perpetuity.

It is made available under a CC-BY 4.0 International license .

indicated by grey dots. B - Receiver operator characteristics (ROC) curve of CD63, HLA-DR and $\mathrm{CD} 11 \mathrm{~b}$ has been plotted. Corresponding area under the curve was observed to be $0.73,0.65$ and 0.63 , respectively. 


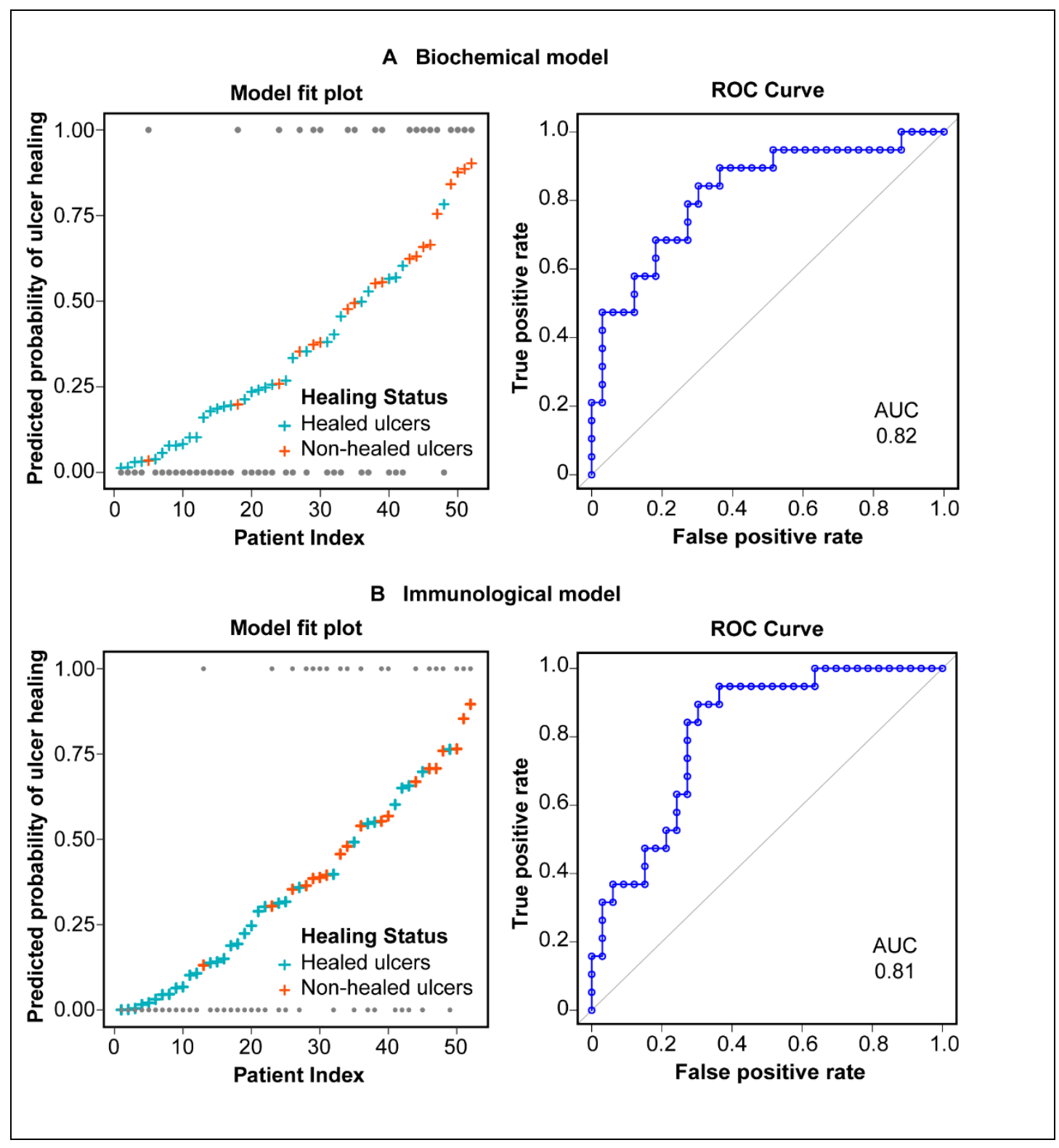

Figure 3: Multivariable Logistic Regression. A and B - Biochemical and cellular parameters (A) and Immunological parameters (B) were used to predict probabilities of healing. The graphs on the left show the fit of the model with reference probabilities indicated as grey dots. The graphs on the right show the receiver operating characteristics (ROC) analysis with area under the curve (AUC) measurements. 


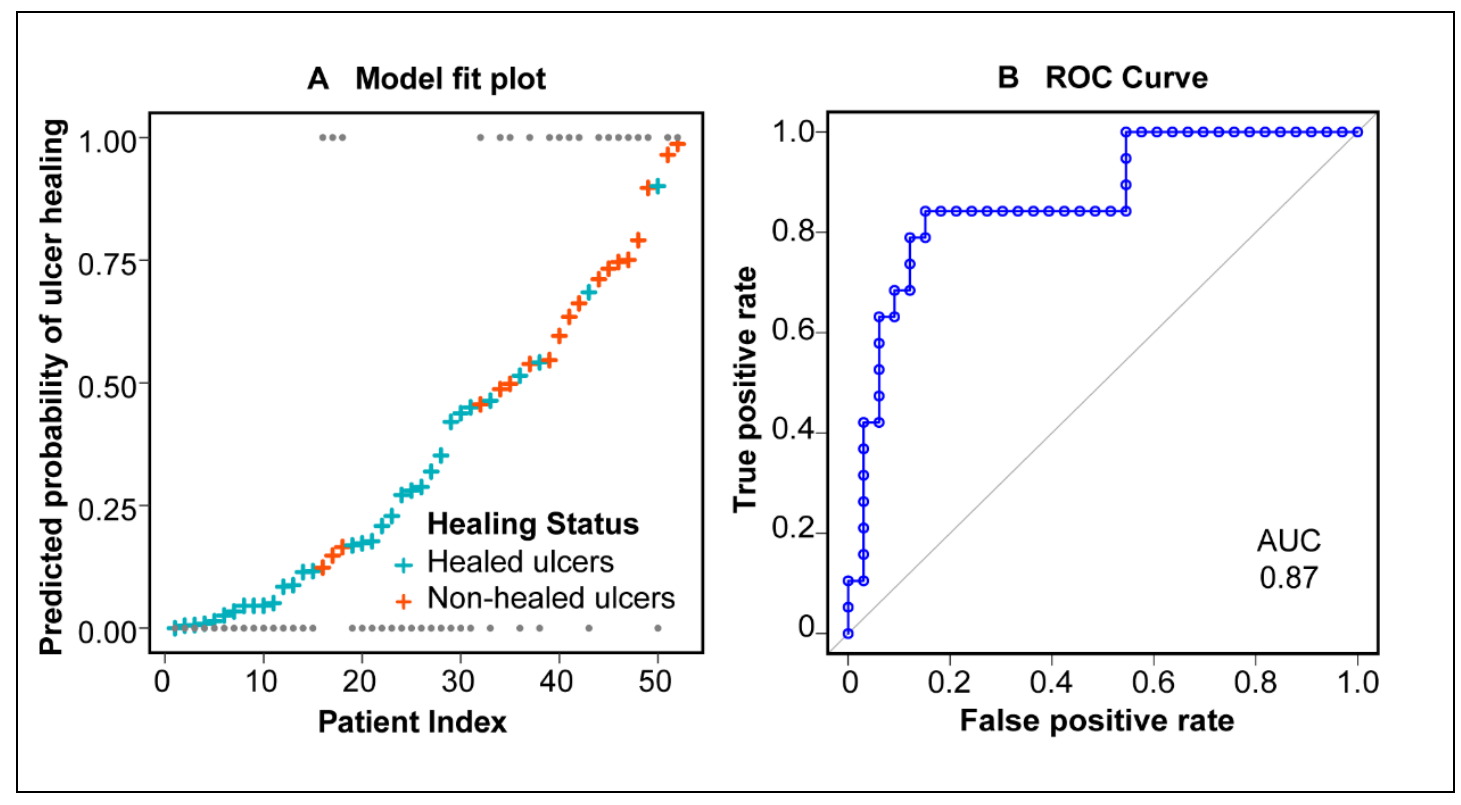

Figure 4: Multivariable Combined logistic regression model performance. A - A

representation of the predicted probabilities of a model, which combines both biochemical and immunological parameters, against reference probabilities (shown by grey dots). B - Receiver operating characteristics (ROC) analysis of combined model shows that the area under the curve (AUC). 